Original

\title{
Prevalencia del síndrome de desgaste profesional (burnout) en odontólogos mexicanos del sector educativo y privado
}

\author{
Prevalence of the syndrome of professional exhaustion (burnout) in mexican \\ dentists of the education sector and private
}

\section{Enrique Castañeda Aguilera, Javier Eduardo García de Alba García} Unidad de Investigación Social, Epidemiológica y de Servicios de Salud (U.I.S.E.S.S.I, Instituto Mexicano del Seguro
Social (I.M.S.S.). Guadalajara, Jalisco, México.

\section{Recibido: 13-07-12}

Aceptado: 01-08-12

\section{Correspondencia:}

Enrique Castañeda Aguilera

Belisario Domínguez № 1000

Col. Independencia 44340 (bajos de la farmacia central)

Centro Médico Nacional de Occidente

Instituto Mexicano del Seguro Social (I.M.S.S.)

Guadalajara. Jalisco (México)

Tel/fax: 36-68-30-00, ext. 31818

E-mail: ecastaneda030@hotmail.com; ecastaneda030@gmail.com

Resumen

Objetivo: Determinar la prevalencia del síndrome de desgaste profesional (burnout) en odontólogos del sector educativo y privado.

Material y métodos: Estudio observacional, descriptivo y transversal, mediante el censo del personal odontológico de la Zona Metropolitana de Guadalajara, México de la Universidad de Guadalajara y los que ejercen la práctica privada, donde participaron 135 de ellos aplicándoseles una ficha de identificación y el Maslach Burnout Inventory-Human Services Survey en forma auto-administrada. Las estadísticas descriptivas y el análisis inferencial se realizaron con apoyo del SPSS 15.0 y Epi-infoV6.1.

Resultados: Se logró el 90,0\% de respuesta. Se detectó una frecuencia del síndrome de desgaste profesional (burnout) en el 50,3\% de ellos. Se obtuvieron diferencias significativas en función a ser menor de 40 años de edad. Se observó la correlación negativa entre las subescalas agotamiento emocional y despersonalización y positiva entre la falta de realización personal en el trabajo.

Conclusiones: El síndrome de desgaste profesional (burnout) es frecuente (50,3\%) en los odontólogos, sus principales factores de riesgo: laborar en la práctica privada, el ser mujer, menor de 40 años de edad, sin pareja estable y con más de 15 años con pareja, el no trabajar la pareja, sin hijos, ser especialista, con menos de 10 años de antigüedad laboral y en el puesto actual de trabajo más de 10 años, laborar en el turno matutino, contratación definitiva y el tener otro trabajo. La afectación del agotamiento emocional y la despersonalización se comporta como el síndrome. Los niveles medios de las subescalas se encuentran en general cerca de la normalidad. Se encontró una correlación negativa entre las subescalas agotamiento emocional y despersonalización y positiva entre la falta de realización personal en el trabajo con la presencia del síndrome. Es necesario establecer medidas preventivas o de intervención en los niveles individual, social u organizacional para reducir la prevalencia encontrada. 
Palabras clave: Odontólogos, desgaste profesional, agotamiento emocional, despersonalización, falta de realización personal.

Abstract

Objective: To determine the prevalence of burnout (burnout) in dental education and private sector.

Material and methods: Methods: Observational, descriptive and cross through the dental staff census metropolitan area of Guadalajara, Mexico at the University of Guadalajara and those in private practice, attended by 135 of them applying to them a form of identification and the Maslach Burnout InventoryHuman Services Survey in a self-administered. Descriptive statistics and inferential analyzes were performed using SPSS 15.0 support and Epi-infoV6.1.

Results: We achieved $90.0 \%$ response. Was detected a frequency of burnout (burnout) in 50.3\% of them. There were significant differences in function to be under 40 years of age. Negative correlation was observed between emotional exhaustion and depersonalization subscales and positive relationship between lacks of personal accomplishment at work.

Conclusions: The syndrome of burnout (burnout) is frequent (50.3\%) for dentists, the main risk factors: work in private practice, being female, under 40 years old without regular partner and with more than 15 years with partner, partner not working, no children, be a specialist, with less than 10 years of work and the current job more than 10 years work in the morning shift, permanent recruitment and have other work. Involvement of the emotional exhaustion and depersonalization behaves like syndrome. The average levels of the subscales are generally near normal. We found a negative correlation between emotional exhaustion and depersonalization subscales and positive relationship between lacks of personal fulfillment in working with the presence of the syndrome. It is necessary to establish preventive or intervention at the individual, social or organizational to reduce the prevalence found.

Med Segur Trab (Internet) 2012; 58 (228) 246-260

Key words: Dentists, burnout, emotional exhaustion, depersonalization, lack of personal accomplishment. 


\section{INTRODUCCIÓN}

La práctica dental ha sido considerada como la que cada vez más en la clínica presenta características y experimenta más problemas de salud física y mental, en comparación con otras profesiones sanitarias. Esto se debe porque ha sido reconocida como una actividad demandante. Para desempeñar sus funciones necesitan gran variedad de equipos y materiales, por lo que están expuestos a sufrir diversos riesgos de trabajo como son accidentes y el estar en contacto con diversos agentes infecciosos, todo esto, aunado a las nuevas tecnologías utilizadas, con aparatos y materiales cada vez más sofisticados que hacen que su labor profesional sea más complicado.

Los factores que inciden en el aumento de la tensión en los odontólogos son: el realizar su actividad laboral en un área limitada, con exigencias de tiempo entre un paciente y otro, las cuestiones ergonómicas como bipedestación prolongada, posturas forzadas y restringidas exclusivamente a la cavidad bucal, en condiciones no óptimas ${ }^{1,2}$, con procedimientos quirúrgicos y dentales prolongados ${ }^{3}$, además de un ambiente de trabajo ruidoso, con insuficiente iluminación y ventilación, así como los factores ambientales que influyen negativamente en la productividad de ellos ${ }^{4}$, una estructura organizacional y un ambiente laboral donde no existe la comunicación entre los mismos trabajadores 5 .

En el mercado laboral del odontólogo existen instituciones de salud perteneciente al estado, por lo que hay pocas vacantes para los servicios dentales y con el incremento acelerado de las demandas de los egresados de las universidades. Ante el escaso número de plazas que es uno de los factores que origina que este opte por realizar la práctica privada. El labor en una institución educativa está condicionada a un horario de trabajo, al que se agrega horas con alumnos en las aulas, preparación de temas teóricos y prácticos, además de la supervisión del alumnado, etc., que difiere del trabajo en forma privada, ya que este adapta sus tiempos de consulta y sus horarios de acuerdo a sus necesidades y las del paciente, el proceso de trabajo se adecua a la situación socioeconómica de estos, por lo que se encuentra determinada por la relación entre la oferta y la demanda de sus servicios. El trabajar con pacientes en el sistema público y privado genera un aumento del nivel de tensión ${ }^{6}$. Todo esto condiciona un nivel de estrés entre estos grupos de profesionales.

Una posible consecuencia del estrés crónico experimentado en el contexto laboral del personal sanitario, incluyendo en él a los odontólogos, es el síndrome de desgaste profesional (burnout) (SDP), que en su forma clínica fue descrito por primera vez en 1974 por el psiquiatra americano Herbert Freudenberger" ${ }^{7}$. Lo definió como "un estado de fatiga o frustración que se produce por la dedicación a una causa, forma de vida o relación que no produce el esperado refuerzo", este fenómeno, que empezó a aparecer en los años setenta en los Estados Unidos de América, para hacer referencia al desgaste o sobrecarga profesional de los profesionales del servicio. En esa misma época la psicóloga Cristina Maslach $^{8}$ estudiaba las respuestas emocionales que se producen entre las personas que trabajaban con los profesionales de ayuda. Y en 1977 dentro del Congreso anual de la Asociación Americana de psicólogos, lo utilizó para describirlo en personas que trabajan en los sectores de servicios humanos, profesionales de la salud y de la educación bajo condiciones difíciles en contacto directo con los usuarios, este término era empleado entonces por los abogados californianos para describir el proceso gradual de pérdida de responsabilidad y desinterés cínico entre compañeros de trabajo.

Maslach y Jackson ${ }^{9}$, lo estudiaron desde una perspectiva tridimensional caracterizado por: agotamiento emocional (AE), manifestado por la pérdida progresiva de energía, cansancio y fatiga; despersonalización (DP), identificada por un cambio negativo de actitudes y respuestas hacia los demás con irritabilidad; y falta de realización personal en el trabajo (RP) con respuestas negativas hacia sí mismo y el trabajo. Y en 1982 establecieron una de las definiciones más aceptadas y utilizadas por los distintos autores para la realización de sus investigaciones: como una "respuesta inadecuada a un estrés emocional 
crónico cuyos rasgos principales son: agotamiento emocional, despersonalización y un sentimiento de inadecuación a las tareas que ha de realizar" ${ }^{10}$. Y en base a los estudios realizados por estas autoras, elaboraron el Maslach Burnout Inventory-Human Services Survey (MBI-HSS), dirigido a los profesionales de la salud. Y validado por Te Brake, Bouman, Gorter, Hoogstraten y Eijkman ${ }^{11}$ en los odontólogos; posteriormente se mostró la versión holandesa del Maslach Burnout Inventory-Nederlandse versie (MBI-NL) desarrollado por Schaufeli y Van Dierendonck ${ }^{12}$ y validado por Gorter, Hoogstraten y Eijkman $^{13}$.

El SDP en odontólogos se considera como un riesgo profesional importante para ellos $^{14}$. Y son propensos al síndrome, debido a la naturaleza específica de su trabajo ${ }^{15}$. En países europeos y americanos se evidencia una prevalencia variable del síndrome, pues las cifras reportadas, oscilan desde el 2-3\% (Alemany, Berini y Gay) ${ }^{16}$ hasta el 50,0\% (Mondol) ${ }^{17}$. Por ejemplo: en hispanoamérica el síndrome se detecta con una frecuencia del 4,5\%; en América del 6,0\% al 50,0\%; en Europa del 2-3\% al 35,7\% y en México del $10,9 \%$ al $36,0 \%$ (tabla I).

Tabla I. Prevalencia del síndrome de desgaste profesional (burnout) (SDP) según diversos autores en odontólogos en el 2012.

\begin{tabular}{ll}
\hline \multicolumn{1}{c}{ Estudio } & \multicolumn{1}{c}{ Resultados } \\
\hline Alemany, Berini y Gay (2008) & $2-3 \%$ en Barcelona, España \\
Gorter, et al. (1999) & $2,5 \%$ en Amsterdam, Holanda \\
Shelley y Wong (1991) & $2,5 \%$ en Amsterdam, Holanda \\
Grau, et al. (2009) & $4,5 \%$ en Hispanoamérica \\
Slate, Steler y Miller (1990) & $6,0 \%$ en Arkansas, Estados Unidos \\
Gorter (2008) & $8,0 \%$ en el Reino Unido \\
Denton, Newton y Bower (2008) & $8,0 \%$ en el Reino Unido \\
Cartagena y Nuñez (2003) & $10,0 \%$ en Talca, Chile \\
Osborne y Croucher (1994) & $10,3 \%$ en el Reino Unido \\
Hernández, et al. (2007) & $10,9 \%$ en Distrito Federal, México \\
Ríos, et al. (2010) & $13,8 \%$ en Sevilla, España \\
Te Brake, et al. (2008) & $15,8 \%$ en Amsterdam, Holanda \\
Gorter y Freeman (2011) & $16,0 \%$ en Irlanda del Norte \\
Peterson, et al. (2008) & $35,7 \%$ en Suecia \\
Díaz; Pérez y Lartigue (2006) & $36,0 \%$ en Distrito Federal, México \\
Marruco (2008) & $38,8 \%$ en Buenos Aires, Argentina \\
Robles y Cáceres (2010) & $44,6 \%$ en Lima, Perú \\
Campos, et al. (2010) & $48,3 \%$ en Sao Paulo, Brasil \\
Mondol (2008) & $50,0 \%$ en San José, Costa Rica \\
Castañeda y García de Alba (2012)1 & $50,3 \%$ en Guadalajara, México \\
\hline Resultados del presente estudio & \\
\hline & \\
\hline
\end{tabular}

Fuente: A partir de las fuentes originales

El conocimiento obtenido hasta la actualidad ha puesto las bases para realizar intervenciones, con apoyo de técnicas y actividades para afrontar y manejar el estrés laboral crónico, todo ello de acuerdo al nivel en el que se intervendrá: individual, grupal o institucional. La mayoría de los programas se centran en el entrenamiento en aspectos 
específicos y, otros, en concientizar a los trabajadores de la importancia de prevenir los factores de riesgo.

Nuestra investigación tiene el objetivo de aportar elementos específicos al estudio del SDP (prevalencia, valores de subescalas y determinación de factores de riesgo), en una muestra censal dentro de una de la instituciones de educación más importantes de América Latina, la Universidad de Guadalajara (UG) y los que ejercen la práctica privada que atienden a la población con patologías dentales, en la zona metropolitana de Guadalajara (ZMG), México.

\section{MATERIAL Y MÉTODOS}

Se realizó un estudio de tipo observacional, descriptivo y transversal con el objeto de determinar la frecuencia y los factores de riesgo del SDP, así como la posible relación con las variables sociodemográficas y laborales asociadas. La población seleccionada fueron los odontólogos que laboran en la UG y los que ejercen la práctica privada en una muestra censal de la ZMG, México, que debían cumplir los siguientes requisitos: más de un año de antigüedad, estar en activo y que tuvieran disposición para contestar los instrumentos de evaluación. Se excluyeron a los odontólogos pasantes (egresados aún no titulados), directivos y demás personal sanitario, así como aquellos que no se encontraban ocupando su plaza en el período de la aplicación de las encuestas, en cuyo caso el cuestionario era complementado por el personal sustituto. Se eliminaron las encuestas que no se llenaron correctamente. Se explicó al personal odontológico los motivos y los objetivos del estudio, obteniéndose su autorización a participar mediante el consentimiento informado verbal haciendo énfasis en que la aplicación sería de carácter anónimo y voluntario, que no conlleva riesgo alguno, hecho lo cual se aplicó.

Para obtener la información se emplearon dos instrumentos: El primero de elaboración propia para el registro de las variables sociodemográficas (sexo, edad, con o sin pareja estable, tiempo con pareja estable, trabaja la pareja, número de hijos y escolaridad) y laborales (práctica laboral, antigüedad laboral y en el puesto actual de trabajo, turno de trabajo, tipo de contratación, otro trabajo y horas que le dedica al mismo). El segundo el MBI-HSS ${ }^{9}$. Utilizándose una versión validada de la original ${ }^{11}$ y en profesionales mexicanos ${ }^{18}$, con un rango de fiabilidad del 0,57 a 0,80 . Se trata de un cuestionario de 22 ítems con 7 opciones de respuesta (escala Likert de 0 a 6), que va de 0 (nunca) hasta 6 (todos los días) contiene las siguientes subescalas: AE ( 9 ítems); DP (5 ítems) y RP (8 ítems). Las puntuaciones de cada subescala se obtienen al sumar los valores de los ítems, lo cual permite evaluar los niveles del síndrome que tiene cada trabajador. Como puntos de corte se pensó en seguir los criterios seguidos por otros autores $^{9,19}$. Para ello se categorizó las tres subescalas en niveles bajo, moderado y alto; AE $(\leq 18 ; 19-26 ; \geq 27)$, DP ( $\leq 5 ; 6-9 ; \geq 10)$, y en contrapartida, la RP, funciona en sentido opuesto a las anteriores $(\leq 33 ; 34-39 ; \geq 40)$, así la puntuación baja, indicarían baja realización, la intermedia, mostraría moderada realización y la alta, como sensación de logro. Determinándose la presencia del SDP con la afectación de una de las subescalas del inventario, ya sea en su nivel medio o alto.

El estudio de los datos y su relación con las variables sociodemográficas y laborales, se realizó mediante el análisis estadístico descriptivo obteniendo cifras absolutas, porcentajes, promedio y desviación estándar de acuerdo a la escala de medición. Para el análisis inferencial de las tablas $2 \times 2$, se aplicó, chi cuadrada (con o sin corrección de Yates), considerando significativa una $\mathrm{p} \leq 0.05$. Se completo el análisis bivariado de las variables, mediante el cálculo del Odds Ratio (OR), con su respectivo intervalo de confianza al 95\%, en el proceso nos apoyamos en los paquetes estadísticos SPSS 15.0 y Epi-info V 6.1. 


\section{RESULTADOS}

De las 150 encuestas distribuidas, cumplieron con los criterios de estudio y se completaron correctamente 133, las 17 restantes de eliminaron por no reunir los pautas establecidas, se obtuvó un porcentaje de respuesta del 88,6\%.

El perfil sociodemográfico: El sexo femenino fue el que destacó con el 51,0\% $(\mathrm{p}<0.05)$, con una edad media de 40,9 $\pm 10,9$ años, el grupo de edad menor a 40 años predominó con el 60,0\% (p<0.05). El 67,0\% (p<0.05) no tenía pareja estable, el tiempo promedio con esta fue del 17,2 $\pm 7,4$ años, el 49,0 \% $(\mathrm{p}<0.05)$ tenía 15 años o más con ella, en el 52,0\% la pareja no trabajaba y el 55,0\% (p<0.05) manifestaron no tener hijos. El 55,0\% presentó un nivel de escolaridad de Especialista $(\mathrm{p}<0.05)$.

El perfil laboral: la antigüedad en la empresa tuvó un promedio de 15,5 \pm 10,3 años, y en el puesto actual de trabajo 11,8 \pm 4,6 años. El grupo de menos de 10 años de antigüedad laboral se mostró en el $55,0 \%(\mathrm{p}<0.05)$ y el grupo de 10 años o más de antigüedad en el puesto actual de trabajo sobresalió con el 54,0\%. El 55,0\% manifestó laborar en el turno matutino, el 51,0\% tenía contratación definitiva $(\mathrm{p}<0.05)$. El 51,0\% tenía otro trabajo y el 52,0\% laboraba más de 4 horas en él $(\mathrm{p}<0.05)$.

El SDP se detectó en 67 (50,3\%) de ellos, de los cuales, $11(16,4 \%)$ tuvieron una subescala afectada, en $26(38,8 \%)$ dos y en $30(54,8 \%)$ tres.

En lo referente a la práctica laboral, la frecuencia del SDP que predominó fue la actividad privada en 34 (25,6\%) de ellos y en la educativa (UG) 33 (24,8\%).

Posteriormente se caracterizaron dos grupos: Uno afectado y otro sin afectar, enseguida se realizó una asociación con cada una de las variables sociodemográficas y laborales.

Obsérvese en la tabla II, que el tener 40 años o más de edad se asoció como variable de riesgo, con la presencia del SDP; comportándose como factor de riesgo el tener 40 años o más de edad [OR = 2,15; $(1,01-4,57)]$.

Tabla II. Relación de las variables socio-demográficas y laborales con la presencia o ausencia de síndrome de desgaste profesional (burnout) en odontólogos de una institución educativa y de la práctica privada de la ciudad de Guadalajara, México $(n=135$ )

\begin{tabular}{|c|c|c|c|c|}
\hline \multirow[t]{2}{*}{ Variables analizadas } & \multicolumn{2}{|c|}{$\begin{array}{l}\text { Síndrome de Desgaste } \\
\text { Profesional (burnout) } \\
\text { (SDP) }\end{array}$} & \multirow[t]{2}{*}{ Valor de $p$} & \multirow[t]{2}{*}{$\begin{array}{l}\text { Significación } \\
\text { estadística }\end{array}$} \\
\hline & $\begin{array}{c}\text { Sí } \\
67(50,3 \%)\end{array}$ & $\begin{array}{c}\text { No } \\
66(49,7 \%)\end{array}$ & & \\
\hline \multicolumn{5}{|c|}{ Perfil sociodemográfico } \\
\hline \multicolumn{5}{|l|}{ Sexo } \\
\hline Masculino & $41(50,0 \%)$ & $41(50,0 \%)$ & \multirow{2}{*}{0,91} & \multirow{2}{*}{ No significativa } \\
\hline Femenino & $26(51,0 \%)$ & $25(49,0 \%)$ & & \\
\hline \multicolumn{5}{|l|}{ Grupo de edad } \\
\hline$<$ De 40 años & $38(60,0 \%)$ & $25(40,0 \%)$ & \multirow{2}{*}{0,029} & \multirow{2}{*}{ Chi $2=4,73$} \\
\hline > De 40 años & $29(41,0 \%)$ & $41(59,0 \%)$ & & \\
\hline \multicolumn{5}{|l|}{ Estado civil } \\
\hline Con pareja estable & $51(47,0 \%)$ & $58(53,0 \%)$ & \multirow{2}{*}{0,12} & \multirow{2}{*}{ No significativa } \\
\hline Sin pareja estable & $16(67,0 \%)$ & $8(33,0 \%)$ & & \\
\hline \multicolumn{5}{|l|}{ Tiempo con pareja estable } \\
\hline$<$ de 15 años & $17(42,0 \%)$ & $23(58,0 \%)$ & \multirow{2}{*}{0,49} & \multirow{2}{*}{ No significativa } \\
\hline$>$ de 15 años & $34(49,0 \%)$ & $35(51,0 \%)$ & & \\
\hline \multicolumn{5}{|l|}{ Trabaja la pareja } \\
\hline No & $26(52,0 \%)$ & $24(48,0 \%)$ & \multirow{2}{*}{0,31} & \multirow{2}{*}{ No significativa } \\
\hline Sí & $25(42,0 \%)$ & $34(58,0 \%)$ & & \\
\hline
\end{tabular}




\begin{tabular}{|c|c|c|c|c|}
\hline Variables analizadas & \multicolumn{2}{|c|}{$\begin{array}{c}\text { Síndrome de Desgaste } \\
\text { Profesional (burnout) } \\
\text { (SDP) }\end{array}$} & Valor de $p$ & $\begin{array}{l}\text { Significación } \\
\text { estadística }\end{array}$ \\
\hline No & $11(55,0 \%)$ & $9(45,0 \%)$ & \multirow{2}{*}{0,65} & \multirow{2}{*}{ No significativa } \\
\hline Sí & $56(49,0 \%)$ & $57(51,0 \%)$ & & \\
\hline \multicolumn{5}{|l|}{ Escolaridad } \\
\hline Profesional & $25(44,0 \%)$ & $32(56,0 \%)$ & \multirow{2}{*}{0,19} & \multirow{2}{*}{ No significativa } \\
\hline Especialidad & $42(55,0 \%)$ & $34(45,0 \%)$ & & \\
\hline \multicolumn{5}{|l|}{ Perfil laboral } \\
\hline \multicolumn{5}{|l|}{ Antigüedad laboral } \\
\hline$<$ de 10 años & $27(55,0 \%)$ & $22(45,0 \%)$ & \multirow{2}{*}{0,40} & \multirow{2}{*}{ No significativa } \\
\hline$>$ de 10 años & $40(48,0 \%)$ & $44(52,0 \%)$ & & \\
\hline \multicolumn{5}{|c|}{ Antigüedad en el puesto actual de trabajo } \\
\hline$<$ de 10 años & $28(48,0 \%)$ & $30(52,0 \%)$ & \multirow{2}{*}{0,67} & \multirow{2}{*}{ No significativa } \\
\hline$>$ de 10 años & $39(54,0 \%)$ & $36(46,0 \%)$ & & \\
\hline \multicolumn{5}{|l|}{ Turno de trabajo } \\
\hline Matutino & $44(55,0 \%)$ & $36(45,0 \%)$ & \multirow{2}{*}{0,19} & \multirow{2}{*}{ No significativa } \\
\hline Vespertino & $23(43,0 \%)$ & $30(57,0 \%)$ & & \\
\hline \multicolumn{5}{|l|}{ Tipo de contratación } \\
\hline Definitiva & $62(51,0 \%)$ & $60(49,0 \%)$ & \multirow{2}{*}{0,73} & \multirow{2}{*}{ No significativa } \\
\hline Temporal & $5(46,0 \%)$ & $6(54,0 \%)$ & & \\
\hline \multicolumn{5}{|l|}{ Otro trabajo } \\
\hline Sí & $25(51,0 \%)$ & $24(49,0 \%)$ & \multirow{2}{*}{0,90} & \multirow{2}{*}{ No significativa } \\
\hline No & $42(50,0 \%)$ & $42(50,0 \%)$ & & \\
\hline \multicolumn{5}{|c|}{ Horas que labora en el otro trabajo } \\
\hline$<$ de 4 horas & $3(43,0 \%)$ & $4(57,0 \%)$ & \multirow{2}{*}{0,64} & \multirow{2}{*}{ No significativa } \\
\hline$>$ de 4 horas & $22(52,0 \%)$ & $20(48,0 \%)$ & & \\
\hline
\end{tabular}

Fuente: Directa, 2012

Se muestran los datos obtenidos para las características de riesgo epidemiológico asociadas a las subescalas de AE (tabla III), DP (tabla IV) y RP (tabla V), con la presencia o no del SDP.

Tabla III. Características de riesgo epidemiológico y estadísticamente asociada con las subescalas de agotamiento emocional (AE) con la presencia o no del síndrome de desgaste profesional (burnout) (SDP) en odontólogos de una institución educativa y de la práctica privada de la ciudad de Guadalajara, México (n = 135)

\begin{tabular}{|c|c|c|c|c|}
\hline \multirow{2}{*}{\multicolumn{2}{|c|}{ VARIABLES }} & \multicolumn{2}{|c|}{$\begin{array}{l}\text { Agotamiento Emocional } \\
\text { (AE) }\end{array}$} & \multirow{2}{*}{$\begin{array}{l}\text { Significación } \\
\text { Estadística }\end{array}$} \\
\hline & & SI (SDP) & NO (SDP) & \\
\hline \multicolumn{5}{|c|}{ PERFIL SOCIODEMOGRÁFICO } \\
\hline \multirow{2}{*}{ Sexo } & Masculino & $26(32 \%)$ & $56(68 \%)$ & \multirow{2}{*}{ No significativa } \\
\hline & Femenino & $16(31 \%)$ & $35(69 \%)$ & \\
\hline \multirow{2}{*}{ Grupo de edad } & $<40$ años & $28(44 \%)$ & $35(56 \%)$ & \multirow{2}{*}{$\begin{array}{c}\text { Chi }^{2}=9,17 \\
\text { p }=0,002^{1}\end{array}$} \\
\hline & $>40$ años & $14(20 \%)$ & $56(80 \%)$ & \\
\hline \multirow{2}{*}{ Estado civil } & $\begin{array}{c}\text { Con pareja estable } \\
\text { (casados/unión libre) }\end{array}$ & $32(29 \%)$ & $77(71 \%)$ & \multirow{2}{*}{ No significativa } \\
\hline & $\begin{array}{c}\text { Sin pareja estable (solteros/ } \\
\text { viudos/divorciados) }\end{array}$ & $10(41 \%)$ & $14(59 \%)$ & \\
\hline \multirow{2}{*}{ Tiempo con pareja estable } & $<15$ años & $7(17 \%)$ & $33(83 \%)$ & \multirow{2}{*}{ No significativa } \\
\hline & $>15$ años & $25(36 \%)$ & $44(64 \%)$ & \\
\hline
\end{tabular}




\begin{tabular}{|c|c|c|c|c|}
\hline \multirow{2}{*}{ VARIABLES } & & \multicolumn{2}{|c|}{$\begin{array}{l}\text { Agotamiento Emocional } \\
\text { (AE) }\end{array}$} & \multirow{2}{*}{$\begin{array}{l}\text { Significación } \\
\text { Estadística }\end{array}$} \\
\hline & & SI (SDP) & NO (SDP) & \\
\hline \multirow{2}{*}{ Trabaja la pareja } & $\mathrm{Si}$ & $15(25 \%)$ & $44(75 \%)$ & \multirow{2}{*}{ No significativa } \\
\hline & No & $17(34 \%)$ & $33(66 \%)$ & \\
\hline \multirow{2}{*}{ Hijos } & $\mathrm{Si}$ & $35(31 \%)$ & $78(69 \%)$ & \multirow{2}{*}{ No significativa } \\
\hline & No & $7(35 \%)$ & $13(65 \%)$ & \\
\hline \multirow{2}{*}{ Escolaridad } & Profesional & $18(32 \%)$ & $39(68 \%)$ & \multirow{2}{*}{ No significativa } \\
\hline & Especialidad & $24(32 \%)$ & $52(68 \%)$ & \\
\hline \multicolumn{5}{|l|}{ PERFIL LABORAL } \\
\hline \multirow{2}{*}{ Práctica laboral } & Educativa (UG) & $18(26 \%)$ & $50(74 \%)$ & \multirow{2}{*}{ No significativa } \\
\hline & Privada & $24(37 \%)$ & $41(63 \%)$ & \\
\hline \multirow{2}{*}{ Antigüedad laboral } & $<10$ años & $20(41 \%)$ & $29(59 \%)$ & \multirow{2}{*}{ No significativa } \\
\hline & $>10$ años & $22(26 \%)$ & $62(74 \%)$ & \\
\hline \multirow{2}{*}{ Antigüedad en el puesto de trabajo } & $<10$ años & $15(26 \%)$ & $43(74 \%)$ & \multirow{2}{*}{ No significativa } \\
\hline & $>10$ años & $27(36 \%)$ & $48(64 \%)$ & \\
\hline \multirow{2}{*}{ Turno laboral } & Matutino & $29(36 \%)$ & $51(64 \%)$ & \multirow{2}{*}{ No significativa } \\
\hline & Vespertino & $13(25 \%)$ & $40(75 \%)$ & \\
\hline \multirow{2}{*}{ Tipo de contratación } & Definitiva & $39(32 \%)$ & $83(68 \%)$ & \multirow{2}{*}{ No significativa } \\
\hline & Temporal & $3(28 \%)$ & $8(72 \%)$ & \\
\hline \multirow{2}{*}{ Otro trabajo } & Sí & $11(22 \%)$ & $38(78 \%)$ & \multirow{2}{*}{ No significativa } \\
\hline & No & $31(37 \%)$ & $53(63 \%)$ & \\
\hline \multirow{2}{*}{ Horas que dedica a otro trabajo } & $<4$ horas & $1(14 \%)$ & $6(86 \%)$ & \multirow{2}{*}{ No significativa } \\
\hline & $>4$ horas & $10(24 \%)$ & $32(76 \%)$ & \\
\hline${ }^{1}$ Asociación significativa. Nivel de signific & estadística $\mathrm{p} \leq 0,05$ & & & \\
\hline
\end{tabular}

Fuente: Directa, 2012

Tabla IV. Características de riesgo epidemiológico y estadísticamente asociada con las subescalas de despersonalización (DP) con la presencia o no del síndrome de desgaste profesional (burnout) (SDP) en odontólogos de una institución educativa y de la práctica privada de la ciudad de Guadalajara, México $(n=135)$

\begin{tabular}{|c|c|c|c|c|}
\hline \multirow{2}{*}{\multicolumn{2}{|c|}{ VARIABLES }} & \multicolumn{2}{|c|}{$\begin{array}{l}\text { Agotamiento Emocional } \\
\text { (AE) }\end{array}$} & \multirow{2}{*}{$\begin{array}{l}\text { Significación } \\
\text { Estadística }\end{array}$} \\
\hline & & SI (SDP) & NO (SDP) & \\
\hline \multicolumn{5}{|c|}{ PERFIL SOCIO DEMOGRÁFICO } \\
\hline \multirow{2}{*}{ Sexo } & Masculino & $38(46 \%)$ & $44(54 \%)$ & \multirow{2}{*}{ No significativa } \\
\hline & Femenino & $19(37 \%)$ & $32(63 \%)$ & \\
\hline \multirow{2}{*}{ Grupo de edad } & $<40$ años & $36(57 \%)$ & $27(43 \%)$ & \multirow{2}{*}{$\begin{array}{c}\mathrm{Chi}^{2}=9,98 \\
p=0,001^{1}\end{array}$} \\
\hline & $>40$ años & $21(30 \%)$ & $49(70 \%)$ & \\
\hline \multirow{2}{*}{ Estado civil } & $\begin{array}{l}\text { Con pareja estable } \\
\text { (casados/unión libre) }\end{array}$ & $44(40 \%)$ & $65(60 \%)$ & \multirow{2}{*}{ No significativa } \\
\hline & $\begin{array}{c}\text { Sin pareja estable (solteros/ } \\
\text { viudos/divorciados) }\end{array}$ & $13(54 \%)$ & $11(46 \%)$ & \\
\hline \multirow{2}{*}{ Tiempo con pareja estable } & $<15$ años & $13(32 \%)$ & $27(68 \%)$ & \multirow{2}{*}{ No significativa } \\
\hline & $>15$ años & $31(45 \%)$ & $38(55 \%)$ & \\
\hline \multirow{2}{*}{ Trabaja la pareja } & $\mathrm{Si}$ & $24(41 \%)$ & $35(59 \%)$ & \multirow{2}{*}{ No significativa } \\
\hline & No & $20(40 \%)$ & $30(60 \%)$ & \\
\hline \multirow{2}{*}{ Hijos } & $\mathrm{Si}$ & $48(42 \%)$ & $65(58 \%)$ & \multirow{2}{*}{ No significativa } \\
\hline & No & $9(45 \%)$ & $11(55 \%)$ & \\
\hline
\end{tabular}




\begin{tabular}{|c|c|c|c|c|}
\hline \multirow[t]{2}{*}{ VARIABLES } & & \multicolumn{2}{|c|}{$\begin{array}{c}\text { Agotamiento Emocional } \\
\text { (AE) }\end{array}$} & \multirow{2}{*}{$\begin{array}{l}\text { Significación } \\
\text { Estadística }\end{array}$} \\
\hline & & SI (SDP) & NO (SDP) & \\
\hline \multirow{2}{*}{ Escolaridad } & Profesional & $20(35 \%)$ & $37(65 \%)$ & \multirow{2}{*}{ No significativa } \\
\hline & Especialidad & $37(49 \%)$ & $39(51 \%)$ & \\
\hline \multicolumn{5}{|l|}{ PERFIL LABORAL } \\
\hline \multirow{2}{*}{ Práctica laboral } & Educativa (UG) & $28(41 \%)$ & $40(59 \%)$ & \multirow{2}{*}{ No significativa } \\
\hline & Privada & $29(45 \%)$ & $36(55 \%)$ & \\
\hline \multirow{2}{*}{ Antigüedad laboral } & $<10$ años & $24(49 \%)$ & $25(51 \%)$ & \multirow{2}{*}{ No significativa } \\
\hline & $>10$ años & $33(39 \%)$ & $51(61 \%)$ & \\
\hline \multirow{2}{*}{ Antigüedad en el puesto de trabajo } & $<10$ años & $24(41 \%)$ & $34(59 \%)$ & \multirow{2}{*}{ No significativa } \\
\hline & $>10$ años & $33(44 \%)$ & $42(56 \%)$ & \\
\hline \multirow{2}{*}{ Turno laboral } & Matutino & $38(47 \%)$ & $42(53 \%)$ & \multirow{2}{*}{ No significativa } \\
\hline & Vespertino & $19(36 \%)$ & $34(64 \%)$ & \\
\hline \multirow{2}{*}{ Tipo de contratación } & Definitiva & $52(43 \%)$ & $70(57 \%)$ & \multirow{2}{*}{ No significativa } \\
\hline & Temporal & $5(45 \%)$ & $6(55 \%)$ & \\
\hline \multirow{2}{*}{ Otro trabajo } & Sí & $21(43 \%)$ & $28(57 \%)$ & \multirow{2}{*}{ No significativa } \\
\hline & No & $36(43 \%)$ & $48(57 \%)$ & \\
\hline \multirow{2}{*}{ Horas que dedica a otro trabajo } & $<4$ horas & $10(67 \%)$ & $5(33 \%)$ & \multirow{2}{*}{ No significativa } \\
\hline & $>4$ horas & $39(63 \%)$ & $23(37 \%)$ & \\
\hline
\end{tabular}

Fuente: Directa, 2012

Tabla V. Características de riesgo epidemiológico y estadísticamente asociada con las subescalas de falta de realización personal en el trabajo (RP) con la presencia o no del síndrome de desgaste profesional (burnout) (SDP) en odontólogos de una institución educativa y de la práctica privada de la ciudad de Guadalajara, México $(n=135)$

\begin{tabular}{|c|c|c|c|c|}
\hline \multirow{2}{*}{\multicolumn{2}{|c|}{ VARIABLES }} & \multicolumn{2}{|c|}{$\begin{array}{c}\text { Falta de Realización } \\
\text { Personal en el Trabajo } \\
\text { (RP) }\end{array}$} & \multirow[t]{2}{*}{$\begin{array}{l}\text { Significación } \\
\text { Estadística }\end{array}$} \\
\hline & & SI (SDP) & NO (SDP) & \\
\hline \multicolumn{5}{|c|}{ PERFIL SOCIODEMOGRÁFICO } \\
\hline \multirow{2}{*}{ Sexo } & Masculino & $14(17 \%)$ & $68(83 \%)$ & \multirow{2}{*}{ No significativa } \\
\hline & Femenino & $9(18 \%)$ & $42(82 \%)$ & \\
\hline \multirow{2}{*}{ Grupo de edad } & $<40$ años & $14(22 \%)$ & $49(78 \%)$ & \multirow{2}{*}{ No significativa } \\
\hline & $>40$ años & $9(13 \%)$ & $61(87 \%)$ & \\
\hline \multirow{2}{*}{ Estado civil } & $\begin{array}{l}\text { Con pareja estable } \\
\text { (casados/unión libre) }\end{array}$ & $16(15 \%)$ & $93(85 \%)$ & \multirow{2}{*}{ No significativa } \\
\hline & $\begin{array}{l}\text { Sin pareja estable (solteros/ } \\
\text { viudos/divorciados) }\end{array}$ & $7(29 \%)$ & $17(71 \%)$ & \\
\hline \multirow{2}{*}{ Tiempo con pareja estable } & $<15$ años & $8(20 \%)$ & $32(80 \%)$ & \multirow{2}{*}{ No significativa } \\
\hline & $>15$ años & $8(12 \%)$ & $61(88 \%)$ & \\
\hline \multirow{2}{*}{ Trabaja la pareja } & $\mathrm{Si}$ & $12(20 \%)$ & $47(80 \%)$ & \multirow{2}{*}{ No significativa } \\
\hline & No & $4(8 \%)$ & $46(92 \%)$ & \\
\hline \multirow{2}{*}{ Hijos } & $\mathrm{Si}$ & $20(18 \%)$ & $93(82 \%)$ & \multirow{2}{*}{ No significativa } \\
\hline & No & $3(15 \%)$ & $17(85 \%)$ & \\
\hline \multirow{2}{*}{ Escolaridad } & Profesional & $9(16 \%)$ & $48(84 \%)$ & \multirow{2}{*}{ No significativa } \\
\hline & Especialidad & $14(18 \%)$ & $62(82 \%)$ & \\
\hline
\end{tabular}




\begin{tabular}{|c|c|c|c|c|}
\hline \multirow[t]{2}{*}{ VARIABLES } & & \multicolumn{2}{|c|}{$\begin{array}{c}\text { Falta de Realización } \\
\text { Personal en el Trabajo } \\
\text { (RP) }\end{array}$} & \multirow[t]{2}{*}{$\begin{array}{l}\text { Significación } \\
\text { Estadística }\end{array}$} \\
\hline & & SI (SDP) & NO (SDP) & \\
\hline \multicolumn{5}{|l|}{ PERFIL LABORAL } \\
\hline \multirow{2}{*}{ Práctica laboral } & Educativa (UG) & $9(13 \%)$ & $59(87 \%)$ & \multirow{2}{*}{ No significativa } \\
\hline & Privada & $14(22 \%)$ & $51(78 \%)$ & \\
\hline \multirow{2}{*}{ Antigüedad laboral } & $<10$ años & $10(20 \%)$ & $39(80 \%)$ & \multirow{2}{*}{ No significativa } \\
\hline & $>10$ años & $13(15 \%)$ & $71(85 \%)$ & \\
\hline \multirow{2}{*}{ Antigüedad en el puesto de trabajo } & $<10$ años & $11(19 \%)$ & $47(81 \%)$ & \multirow{2}{*}{ No significativa } \\
\hline & $>10$ años & $12(16 \%)$ & $63(84 \%)$ & \\
\hline \multirow{2}{*}{ Turno laboral } & Matutino & $13(16 \%)$ & $69(84 \%)$ & \multirow{2}{*}{ No significativa } \\
\hline & Vespertino & $10(19 \%)$ & $43(81 \%)$ & \\
\hline \multirow{2}{*}{ Tipo de contratación } & Definitiva & $22(18 \%)$ & $100(82 \%)$ & \multirow{2}{*}{ No significativa } \\
\hline & Temporal & $1(9 \%)$ & $10(91 \%)$ & \\
\hline \multirow{2}{*}{ Otro trabajo } & Sí & $9(18 \%)$ & $40(82 \%)$ & \multirow{2}{*}{ No significativa } \\
\hline & No & $14(17 \%)$ & $70(83 \%)$ & \\
\hline \multirow[b]{2}{*}{ Horas que dedica a otro trabajo } & $<4$ horas & $7(54 \%)$ & $6(46 \%)$ & \multirow{2}{*}{$\begin{array}{c}\mathrm{Chi}^{\mathbf{2}} \text { con } \\
\text { corrección de } \\
\text { Yates }=\mathbf{4}, \mathbf{4 3} \\
\mathbf{p}=\mathbf{0 , 0 3}\end{array}$} \\
\hline & $>4$ horas & $8(19 \%)$ & $34(81 \%)$ & \\
\hline${ }^{1}$ Asociación significativa. Nivel de s & Ición estadística & 0,05 & & \\
\hline
\end{tabular}

Fuente: Directa, 2012

Se asociaron como variables de riesgo, con la presencia del SDP, para la subescala AE y DP el tener menos de 40 años de edad; para la RP el laborar menos de 4 horas en otro trabajo. Comportándose como factor de riesgo: para AE menos de 40 años de edad [OR = 3,20; (1,39 - 7,43)]; para la DP el tener menos de 40 años de edad [OR =3,11; $(1,44$ - 6,79)] y para la RP laborar menos de 4 horas en otro trabajo [OR $=6,06 ;(1,09-23,55)$ ].

Se efectuó una correlación con SDP en las 3 subescalas (no referidas en las tablas), presentando una asociación inversamente proporcional del AE $(-0.624)(\mathrm{p}=0,0001)$ y DP $(-0.8004)(\mathrm{p}=0,0001)$, y directamente proporcional de la RP $(0.810)(\mathrm{p}=0,0001)$.

En la tabla VI, se establecieron los valores medios en las subescalas de AE $(15,0)$, DP $(5,9)$ y RP $(36,9)$ de nuestro estudio comparándolo con diversos autores.

Tabla VI. Valores medios en las subescalas de agotamiento emocional (AE), despersonalización (DP) y la falta de realización personal en el trabajo (RP) del síndrome de desgaste profesional (burnout) (SDP) en odontólogos, según diversos autores en el 2012.

\begin{tabular}{lccc}
\hline & $\begin{array}{c}\text { Agotamiento } \\
\text { emocional (AE) }\end{array}$ & $\begin{array}{c}\text { Despersonalización } \\
\text { (DP) }\end{array}$ & $\begin{array}{c}\text { Falta de realización } \\
\text { personal en el trabajo } \\
\text { (RP) }\end{array}$ \\
\hline Valores normales (Maslach y Jackson, 1986) & $\mathbf{2 2 , 1}$ & $\mathbf{7 , 2}$ & $\mathbf{3 6 , 5}$ \\
Alpöz (2008) & 11,9 & 4,2 & 24,1 \\
Gorter, et al. (1999) & 13,7 & 5,9 & 30,8 \\
Castañeda y García de Alba (2012) & $\mathbf{1 5 , 0}$ & $\mathbf{5 , 9}$ & $\mathbf{3 6 , 9}$ \\
Oliveira y Slavutzky (2001) & 16,6 & 2,8 & 43,6 \\
Albanesi, et al. (2005) & 17,0 & 6,0 & 41,7 \\
Maslach y Jackson (1985) & 20,9 & 8,7 & 34,5
\end{tabular}




\begin{tabular}{lccc}
\hline & $\begin{array}{c}\text { Agotamiento } \\
\text { emocional (AE) }\end{array}$ & $\begin{array}{c}\text { Despersonalización } \\
\text { (DP) }\end{array}$ & $\begin{array}{c}\text { Falta de realización } \\
\text { personal en el trabajo } \\
\text { (RP) }\end{array}$ \\
\hline Martínez (2009) & 21,6 & 5,6 & 38,6 \\
Grau, et al. (2009) & 21,7 & 5,7 & 39,2 \\
Gorter y Freeman (2011) & 24,3 & 8,6 & 33,0 \\
Osborne y Croucher (1994) & 25,5 & 8,8 & 34,4 \\
Varela, et al. (2005) & 26,1 & 10,7 & 33,1 \\
\hline
\end{tabular}

1 Resultados del presente estudio

Fuente: A partir de las fuentes originales

\section{DISCUSIÓN}

El estudio del SDP ha ido creciendo en complejidad a medida que se ha profundizando en la identificación y evaluación de sus posibles causas, determinantes y procesos adyacentes. El estudio de éste en una muestra representativa de odontólogos de la ZMG, en México, tiene como fortaleza, aportar elementos de juicio no tan solo para constatar la creciente importancia de la magnitud del síndrome en el área de la salud y la afectación del personal sanitario, sino también describir y analizar factores de riesgo y de protección asociados en un medio educativo y privado, siendo una de sus principales modalidades en la atención a la población con y sin acceso a la seguridad social.

Las principales restricciones del trabajo, están relacionadas con su diseño transversal y con algunas propiedades del instrumento de recolección de datos utilizado, el cuestionario estructurado, que disminuye las respuestas obtenidas de los sujetos de estudio a las opciones empleadas que limitan la explicación o profundización del tema investigado. Otras posibilidades fueron: el sesgo de memoria y la reserva individual por temor a que la información otorgada se usara de forma negativa. El primer aspecto se solvento al utilizar cuestionarios validados, el segundo se trato de evitar mediante el uso de preguntas concretas y objetivas y el tercero al asegurar el anonimato de los informantes.

Lo anterior se logró en nuestro estudio, con el 88,6 \% de respuesta, que aunado al tamaño de la muestra, para que ofrezca representatividad en relación a los odontólogos que laboran en el contexto educativo y privado de la ZMG, México y que reflejó una mayor frecuencia que los otros estudios reportados en la literatura (del 50,0\% al 76,1\%) ${ }^{11 \text {, }}$ $13,14,20-32$.

En lo referente al perfil sociodemográfico: El sexo femenino fue el que destacó en nuestro estudio que concuerda con la literatura médica revisada ${ }^{16,21,27,29,30,34-40}$. No

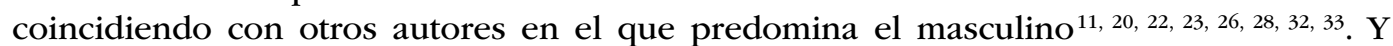
Hernández, et al., en el $2007^{41}$ encontró la misma proporción en ambos. Aunque Te Brake, et al., en el $2003^{32}$ concluyen en su investigación que la diferencia de género en el SDP entre los dentistas no existe. La edad también fue analizada, en donde el mayor número de casos se presentó en el grupo menor de 40 años, que se ajusta a las investigaciones revisadas ${ }^{16,27,29,40,41}$ y se asoció significativamente. No coincidiendo con otros autores ${ }^{15,20,21,23,26,30,32,35,36,38}$ en que predominaron las personas de más de 40 años. El no tener pareja estable se mostró con mayor frecuencia en nuestro estudio, que coincide con las investigaciones de Alemany, et al., en el $2008^{16}$ y Hernández, et al., en el $2007^{41}$; aunque en otros estudios prevaleció el tener pareja estable ${ }^{21,27,28,32,37,40}$. En nuestra investigación resaltó el tener 15 años o más con ella, también se manifestó el que la pareja no trabajaba, ambas no fueron reportadas en los estudios revisados. El no tener hijos sobresalió con nosotros, no coincidiendo con otros autores ${ }^{16,21,27}$ en donde se mostró el poseerlos. La escolaridad que sobresalió fue el tener especialidad que concuerda con 
los estudios examinados ${ }^{16,21,27,28,33,40}$; no obstante para otros investigadores ${ }^{29,41}$ se mostró la mayor frecuencia en ser profesionista.

En lo concerniente al perfil laboral: El tener una antigüedad laboral menor a 10 años se destacó en nuestra investigación, que coincide con los estudios realizados por Al-Mobberiek y Al-Mobberiek en el $2011^{29}$ y Hernández, et al., en el $2007^{41}$; la antigüedad en el puesto actual de trabajo de 10 años o más sobresalió y concuerda con varios autores $^{20,21,35,36}$, en otras investigaciones se hallaron niveles alarmantes en las etapas iniciales de su vida profesional ${ }^{23,24}$, para Odrich y Wayman en $1987^{42}$ encontraron que a mayor antigüedad laboral esta es predictiva de presentar niveles elevados del síndrome. En lo referente al turno laboral resaltó el matutino, que no fueron reportados en los estudios examinados. El tipo de contratación definitiva fue el que predominó en nuestro estudio, no coincidiendo con lo realizado por Varela, et al., en el $2005^{20}$ en el que sobresalió la contratación temporal. El tener otro empleo resaltó coincidiendo con la investigación de Aparicio, en el $2008^{21}$.

Para contextualizar nuestro estudio, se hizo una amplia revisión sobre la prevalencia del SDP en odontólogos (tabla I), que nos ubica por encima de todas las investigaciones revisadas $^{11,16,26,28,30,34-37,41,43-51}$ evidenciando la amplia variabilidad en la frecuencia del síndrome, situación que a su vez pone de manifiesto el carácter complejo de sus componentes.

La práctica laboral que predominó fue el trabajar en el sector privado que es concordante con los estudios realizados por Ríos, et al., en el $2010^{28}$ y De Oliveira y Slavutzky, en el $2001^{40}$. Y para Gorter y Freeman, $2011^{30}$ se destacó en ambas prácticas. Para Rutter, et al., en el $2002^{51}$ el asumir el doble rol, tanto educativo como clínico, ambos pueden incrementar los niveles del síndrome, pero también hay evidencias que esta doble función puede reducirlo.

Se nota un rasgo de riesgo consistente para él: SDP, AE y DP juntos son: tener menos de 40 años de edad.

Los valores medios de la subescalas AE se encuentran dentro de los parámetros bajos, la DP y la RP se encuentran las puntuaciones dentro del nivel medio de la normalidad (tabla VI).

El establecimiento de una correlación negativa con la presencia del SDP entre las subescalas AE y DP y positiva con la de RP, está en concordancia con lo encontrado por Alpöz, et $a l^{27}$, y Salanova, et $a l^{52}$. Este resultado también ha sido confirmado mediante modelos de ecuaciones estructurales ${ }^{53,54}$. Se confirma que el AE es la subescala más fiable del síndrome. Schaufeli y Enzmann ${ }^{55}$ mencionan que las dos dimensiones centrales del síndrome son el AE y la DP y son el corazón de éste ${ }^{56}$.

Por lo que se concluye que el SDP es frecuente $(50,3 \%)$ en los odontólogos, sus principales factores de riesgo: laborar en la práctica privada, el ser mujer, menor de 40 años de edad, sin pareja estable y con más de 15 años con pareja, el no trabajar la pareja, sin hijos, ser especialista, con menos de 10 años de antigüedad laboral y en el puesto actual de trabajo más de 10 años, laborar en el turno matutino, contratación definitiva y el tener otro trabajo. La afectación del AE y la DP se comporta como el síndrome. Los niveles medios de las subescalas se encuentran en general cerca de la normalidad. Se encontró una correlación negativa entre las subescalas agotamiento emocional y despersonalización y positiva entre la falta de realización personal en el trabajo con la presencia del síndrome. Lo anterior nos lleva a considerar la necesidad de establecer medidas preventivas o de intervención en los niveles individual, social u organizacional para reducir la prevalencia encontrada. 


\section{REFERENCIAS BIBLIOGRÁFICAS}

1. Wycoff S. An examination of what dentists already know about stress and burnout within Dentistry. CDA Journal 1984; 12 (12): 114-117.

2. Pollack R. Dental office ergonomics: How reduce stress factors and increase efficiency. J Can Dent Assoc 1996; 62 (6): 508-510.

3. Freeman R, Main J, Burke F. Occupational stress and Dentistry: theory and practice Part I Recognition. Brit Dent J 1995; 178 (6): 214-217

4. Romero R, Becerra T, Velasco M. Síndrome de Burnout. Desgaste emocional en cirujanos dentistas. Revista ADM 2001; 58 (2): 63-67.

5. Turley Kinirons M, Freeman R. Occupational stress factors in hospital dentists. Brit Dent J 1993; 175 (8): 285-288.

6. Newton J, Gibbons D. Stress in dental practice: a qualitative comparison of dentists working within the NHS and those working within an independent capitation scheme. Brit Dent J 1996; 180: (9); 329-334.

7. Freudenberger M. Staff Burn-out. J Soc Issues 1974; 30: 159-166.

8. Maslach C. Burned out. Hum Behavior 1976; 59: 16-22.

9. Maslach C, Jackson S. Maslach Burnout Inventory. Manual. Palo Alto, California: Consulting Psychologist Press, 1981.

10. Maslach C. Understanding Burnout definitional issues in analyzing a complex phenomenon. En: Paine: WS. Editor Job, Stress and Burnout. Beverly Hills: Sage Pub. 1982.

11. Te Brake, J, Bouman A, Gorter R, Hoogstraten J, Eijkman M. Using the Maslach Burnout Inventory among dentists: burnout measurement and trends. Community Dent and Oral Epidemiol 2008; 36 (1); 69-75.

12. Schaufeli W, Van Dierendonck D. Maslach Burnout Inventory, Nederlandse versie (MBI-NL). Provisional manual. Utrecht the Netherlands: Utrecht University, Research School Psychology \& Health, 1995.

13. Gorter R, Albrecht G, Hoogstraten J, Eijkman M. Factorial validity of the Maslach burnout inventoryDutch version (MBI-NL) among dentists. J Organiz Behav 1999; 20 (2): 209-217.

14. Gorter R, Hoogstraten J, Eijkman M, Te Brake H. Type of dentist, career expectancies, and burnout among Dutch dentists. Amsterdam, Netherlands. University of Amsterdam, Netherlands. Abstract no. 1310. 2002.

15. Purine A, Janulyte V, Musteikyte M, Bendinskaite R. General Health of Dentists. Literature Review. Stomatologija 2007; 9: 10-20.

16. Alemany A, Berini L, Gay C. The burnout syndrome and associated personality disturbances. The study in three graduate programs in Dentistry at the University of Barcelona. Med Oral Patol Oral Cir Bucal 2008; 13 (7): E444-E450.

17. Mondol M. Análisis de la prevalencia del Síndrome de Burnout en los odontólogos y sus repercusiones en la salud del profesional y en la atención bucodental del Servicio de Odontología en la Clínica Carlos Durán durante el periodo agosto-noviembre de 2007. Programa de Maestría Nacional en Gerencia de la Salud. Instituto Centroamericano de Administración Pública ICAP. 2008.

18. Grajales G. Estudio de la validez factorial del Maslach Burnout Inventory versión Española en una población de profesionales mexicanos. Línea de Investigación: Instrumentos de medición SELA 2001: $1-10$.

19. De las Cuevas C. El desgaste profesional en atención primaria: presencia y distribución del síndrome de burnout. MAFRE Medicina 1994; 6: 7-18.

20. Varela P, Fontao L, Martínez A, Pita A, Valín M. Desgaste profesional entre los odontólogos y estomatólogos del Servicio Gallego de Salud. Aten Primaria 2005; 35 (6): 301-305.

21. Aparicio S. Prevalencia del Síndrome de Burnout en docentes de Odontología del Colegio Odontológico de la Institución Universitaria Colegio de Colombia en la ciudad de Bogotá. Journal Odontológico Colegial 2008; 2: 71-77.

22. Te Brake, J, Bouman A, Gorter R, Hoogstraten J, Eijkman M. Professional burnout and Work engagement among dentists. Eur J Oral Sci 2007; 115 (3): 180-185.

23. Gorter R, Storm M, Eijkman M. Burnout measurements in early dental career. Amsterdam, Netherlands, abstract no. 2613, 2004.

24. Gorter R, Storm M, Te Brake J, Kersten H, Eijkman M. Outcomes of career experiencies and professional burnout among newly qualified dentists. Int Dent J 2007; 57 (4): 279-285. 
25. Gorter R, Eijkman M, Hoogstraten J. Burnout and Health among Dutch dentists. Eur J Oral Sci $2000 ; 108$ (4): 261-267.

26. Gorter R, Albrecht G, Hoogstraten J, Eijkman M. Professional burnout among Dutch dentists. Community Dent and Oral Epidemiol 1999; 27(2): 109-116.

27. Alpöz E, Güneri P, Sürgevil O, Cankaya H. Burnout Syndrome in A Dentistry Faculty: Effect of Sociodemographic and Academic. ARASTIRMA (Research) 2008; 32 (3): 18-28.

28. Ríos J, Reyes M, López A, Morillo J, Bullón P. Burnout and depression among Spanish periodontology practitioners. Med Oral Patol Oral Cir Bucal 2010; 15 (5): e813-e819.

29. Al-Mobberiek H, Al-Mobberiek A. Burnout among dental academics and no-academics in Riyadh and Eastern Province, Saudi Arabia. J Pak Dent Assoc 2011; 20(4): 199-205.

30. Gorter R, Freeman R. Burnout and engagement in relation with job demands and resources among dental staff in Northern Ireland. Community Dent Oral Epidemiol 2011; 39: 87-95.

31. Gorter R, Albrecht G, Hoogstraten J, Eijkman M. Work place characteristics work stress and burnout among Dutch dentists. Eur J Oral Sci 1998; 106 (6): 999-1005.

32. Te Brake H, Bloemendal E, Hoogstraten J. Gender differences in burnout among Dutch dentists. Community Dent Oral Epidemiol 2003; 31 (5): 321-327.

33. Martínez A. Estudio sobre 1 síndrome de desgaste profesional en Odontología. Gaceta Dental 2003; 143: 38-51.

34. Robles R, Cáceres L. Síndrome Burnout en cirujanos dentistas de hospitales de Lima Metropolitana. Odontología Sanmarquina 2010; 13(1): 7-10.

35. Campos J, Trotta O, Bonafé F, Maroco J. Burnout em dentistas do serviço público: ter ou não ter, eis a questão! Rev Odontol UNESP, Araraquara 2010; 39(2): 109-114.

36. Peterson U, Barnstorm G, Demerouti E, Asberg M, Nygren A. Work characteristics and sickness absence in burnout and non-burnout groups: A study of Swedish health care workers. International Journal of Stress Management 2008; 15(2): 153-172.

37. Díaz R, Pérez E, Lartigue M. Desgaste profesional en un grupo de odontólogos. Revista ADM 2006; 63 (6): 220-224.

38. Zaragoza S, Pozos B, Muñoz H, López S. Burnout en los odontólogos de la Secretaria de Salud de Jalisco, México. La Habana, Cuba. Abstract no. 25. 2007.

39. Murtomaa H, Haavio-Mannila E, Kandolin I. Burnout and its causes in Finnish dentists. Community Dent Oral Epidemiol 1990; 18: 208-212.

40. Oliveira R, Slavutzky S. A sindrome de Burnout nos cirurgiões-dentistas de Porto Alegre, RS. Rev Fac Odontol Porto Alegre 2001; 43 (2): 45-50.

41. Hernández C, Monroy A, Rivera P, Rojas B, Sánchez N. Estudio comparativo de los síntomas del Síndrome de Burnout entre Odontólogos de la práctica general y especialista del Distrito Federal y área metropolitana en el 2007. México: Universidad Nacional Autónoma de México. Facultad de Estudios Superiores Iztacala, módulo de laboratorio II; 2007.

42. Odrich J, Wayman D. Institutional setting and burnout levels in dental hygiene educators. Dent Hygiene 1987; 61 (2): 74-78.

43. Gorter R. Occupational burnout and work engagement: a national survey of dentists in the United Kingdom. Brit Dent J 2008; 205: 382-383.

44. Cartagena H, Núñez M. Síndrome de Burnout en odontólogos del nivel primario de atención adscritos a la dirección comunal de salud de Talca. Talca: Universidad de Talca; 2003.

45. Mondol M. Análisis de la prevalencia del Síndrome de Burnout en los odontólogos y sus repercusiones en la salud del profesional y en la atención bucodental del Servicio de Odontología en la Clínica Carlos Durán durante el periodo agosto-noviembre de 2007. Programa de Maestría Nacional en Gerencia de la Salud. Instituto Centroamericano de Administración Pública ICAP. 2008.

46. Marruco M. Síndrome de quemarse por el trabajo (burnout) en odontólogos residentes en seis hospitales de la Provincia de Buenos Aires. Rev Asoc Odontol Argent 2008; 96 (1): 49-52.

47. Denton D, Newton J, Bower E. Occupational burnout and work engagement: a national survey of dentists in the United Kingdom. Brit Dent J 2008; 205: 382-383.

48. Grau A, Flichtentrei D, Suñer R, Prats M, Braga F. Influencia de factores personales, profesionales y transnacionales en el Síndrome de Burnout en personal sanitario hispanoamericano y español 2007. Rev Esp Salud Pública 2009; 83(2): 215-230.

49. Osborne D, Croucher R. Levels of burnout in general dental practitioners in the south-east of England. Brit Dent J 1994; 177 (10): 372-377. 
50. Shelley J, Wong M. Prevalence of burnout among military dentists. Milit Med 1991; 156 (3): 113-118

51. Rutter H, Herzberg J, Paice E. Stress in doctors and dentists who teach. Med Educ 2002; 36 (6): $543-549$.

52. Salanova M, Schaufeli W, Llorens S, Peiró J, Grau R. Desde el "burnout” al "Engagement” ¿Una nueva perspectiva? Rev Psicol Trab Organ 2000; 16:117-134.

53. Schaufeli W, Martínez I, Marqués A, Salanova M, Bakker A. Burnout and engagement in university students: A cross-national study. J Cross Cult Psychol 2002; 33: 464-481.

54. Manzano G. Bienestar subjetivo de los cuidadores formales de Alzheimer: relaciones con el burnout, engagement y estrategias de afrontamiento. Ansiedad y Estrés 2002; 8: 225-244.

55. Schaufeli W, Enzmann D. The burnout companion to study and research: A critical analysis. London: Taylor and Francis, 1998.

56. Lee R, Ashforth B. A meta-analytic examination of the correlates of the three dimensions of job burnout. J Appl Psychol 1996; 81(2): 123-133.

|||||||||||||||||||||||||||||||||||||||||||||||||||||||||||||||||||||||||||||||||||||||||||||||||||||||||||||||||||||||||||||||||||||||||||||||||||||||||||||||||||||||||||||||||||||||||||||||||||||||| 